\title{
OS CARVALHAIS DO PARQUE NATURAL DAS SERRAS DE AIRE E CANDEEIROS (CENTRO DE PORTUGAL). SUA CONSERVAÇAO
}

\author{
Mário FERNANDES LOUSA \& Maria Dalila ESPIRITO SANTO
}

\begin{abstract}
RESUMO: Os carvalhais do Parque Natural das Serras de Aire e Candeeiros no Centro-Oeste de Portugal, são aqui estudados pelos métodos da escola de Zurich-Montpellier e dos perfis ecológicos e informação mútua. Os resultados obtidos permitem afirmar que as espécies mais sensíveis à dominância de Quercus faginea Lam. ssp. broteroi (P. Coutinho) A. Camus estão incluídas essencialmente na associação Arisaro clusii-Quercetum broteroi $\mathrm{Br}$.-B1., P. Silva \& Rozeira, 1956, nos sintáxones de hierarquia superior ou nas comunidades da mesma série de vegetação.
\end{abstract}

Palavras chave: Fitossociologia, Quercus faginea ssp. broteroi, Portugal.

SUMMARY: The oakwoods of Serras de Aire e Candeeiros Natural Park in Portugal CenterWest are studied here by the methods of Zurich-Montpellier school and ecological profiles and mutual information. The results allow to state that the most sensitive species to Quercus faginea Lam. ssp. broteroi (P. Coutinho) A. Camus domination are essencially included in Arisaro clusii-Quercetum broteroi Br.-Bl., P. Silva \& Rozeira, 1956, association, in superior hierarchy sintaxa or in the communities of the same vegetation serie.

Key words: Phytosociology, Quercus faginea, ssp. broteroi, Portugal.

\section{N T R O D U Ç Ã O}

O Parque Natural das Serras de Aire e Candeeiros situa-se no Centro-Oeste calcário de Portugal. É constituído geologicamente essencialmente por formações do Jurássico Médio.

A Serra dos Candeeiros orienta-se quase na direcção Norte-Sul ao longo de uma faixa de $30 \mathrm{Km}$ cujo ponto culminante atinge os $613 \mathrm{~m}$. A serra de Aire está situada a Sueste de Fátima e é um pouco mais alta elevando-se até $679 \mathrm{~m}$ de altitude. Neste Parque existem numerosas grutas algumas de grande dimensão e extensão.

Só uma parte diminuta desta Reserva, nos vales e depressões fechadas onde se acumulou um solo fértil, se encontra cultivada. Quase todo o resto está coberto de matos, matagais e olivais abandonados ou semi-abandonados. Apenas algumas áreas 
exíguas e muito localizadas estão cobertas por carvalhais de Quercus faginea Lam ssp. broteroi (P. Coutinho) A. Camus. Além desta espécie aparece Quercus pyrenaica Willd. reduzido a alguns exemplares na zona sul do Parque e o híbrido Quercus xairensis Franco \& Vasc., arbustivo alto que se encontra na parte central da Reserva.

Segundo Albuquerque (1978) as precipitações variam de $1000 \mathrm{~mm}$ na zona de Porto de Mós a $1500 \mathrm{~mm}$ na zona de maior altitude. A temperatura média do mês mais frio em qualquer ponto do Parque é superior a $5^{\circ} \mathrm{C}$ estando a média das mínimas do mês mais frio compreendida entre 0 e $3^{\circ} \mathrm{C}$. $\mathrm{O}$ andar bioclimático de Emberger correspondente ao Parque é o húmido de inverno fresco (Alcoforado et al., 1982). De acordo com Rivas-Martínez (1987) o piso bioclimático deste Parque é o Mesomediterrâneo em transição para o Termomediterrâneo; a região em estudo sob o ponto de vista biogeográfico pertence à Região Mediterrânea, subregião Mediterrânea ocidental, superprovíncia Mediterrâneo-Iberoatlântica, Província Luso-Extremadurense, Sector Divisório Português.

A vegetação clímax é constituída por bosques geralmente mais ou menos abertos de Quercus faginea Lam. ssp. broteroi (P. Coutinho) A. Camus que podem ser localizados em pequenas manchas nos vales e a meia encosta. No cimo das serras apareceriam bosques de Quercus rotundifolia Lam. ainda imagináveis pela vegetação subarbustiva repetidamente destruída pelos fogos. Por degradação dos bosques surgem formações arbustivas altas de Quercus coccifera L. geralmente na zona superior das encostas. Por degradação ainda maior poder-se-ão ver matos baixos onde podem predominar Cistus albidus L., C. monspeliensis L., C. salvifolius L., Thymus zygis L. ou ainda Rosmarinus officinalis L., resultado da acção intensiva do fogo e do pastoreio.

É fundamental a renovação dos bosques, neste caso de $Q$. faginea Lam. ssp. broteroi (P. Coutinho) A. Camus. O ambiente fresco e agradável, a diversidade biológica, a riqueza dos estratos inferiores, a manta morta que actua como esponja nas chuvas invernais evitando a erosão, a riqueza biológica do solo, são aspectos a ter em conta quando se avaliam estas formações. Normalmente situam-se em locais onde dificilmente outro tipo de vegetação se desenvolve com tanta facilidade - zonas declivosas com afloramentos rochosos. Em condições propícias pode o Quercus faginea Lam. ssp. broteroi (P. Coutinho) A. Camus atingir grande porte podendo formar belos bosques e ter interesse para o corte quando efectuado criteriosamente.

\section{A T ER I A L E M ÉTODOS}

Em 1979/1980 realizou-se o estudo florístico do Parque Natural das Serras de Aire e Candeeiros (Espírito Santo \& Lousã, 1981) mediante a transcrição das condições ambientais e do elenco florístico para fichas apropriadas (IFs) repondo o método da Escola de Zurich-Montpellier (Braun-Blanquet, J. 1932).

Em 1986 para aplicação do método dos perfis ecológicos e da informação mútua (Daget \& Godron, 1982), acrescentaram-se aos inventários realizados mais dados ecológicos que foram codificados com base no código do CEPE (Godron et al., 1968). dando origem a um ficheiro ecológico e outro fitossociológico. O tratamento dos dados foi feito em computador VAX/VMS mediante um conjunto de programas 
(Lepart, 1982; Bacou et Lepart, 1984) cedido pelo "Centre d'Études Phytosociologiques et Écologiques Louis Emberger" (CNRS) de Montpellier.

\section{R E S UL T A D O S}

Os inventários fitossociológicos trabalhados segundo os métodos da Escola de Zurich-Montpellier, desenvolvida por Braun-Blanquet (1932) e actualizada por Géhu e Rivas-Martínez (1980) são apresentados num quadro fitossociológico definitivo em que as espécies vêm distribuídas pelos vários sintáxones aos quais estão mais ligados.

Os carvalhais do Parque Natural das Serras de Aire e Candeeiros pertencem à associação Arisaro clusii-Quercetum broteroi Br.-B1., P. Silva \& Rozeira, 1956. A riqueza em espécies arbustivas está relacionada com o seu corte e mesmo arranque a intervalos regulares.

O elenco florístico destas formações arbóreas de $Q$. faginea Lam. ssp. broteroi (P. Coutinho) A. Camus mostra uma riqueza específica de elementos das classes Querco-Fagetea Br.-Bl. \& Vlieger 1937 e Trifolio-Geranietea sanguinei Th. Muller 1962. Esta última é formada pela vegetação herbácea vivaz que se radica nas clareiras e orlas de bosques espontâneos geralmente caducifólios, como acontece nestes carvalhais. Querco-Fagetea engloba espécies que encontram o seu óptimo em bosques caducifólios onde encontram condições de frescura, sombreamento e humidade edáfica (Franzi 1984) apropriada, condições estas que se podem encontrar nos carvalhais de Quercus faginea Lam. ssp. broteroi (P. Coutinho) A. Camus do Parque Natural das Serras de Aire e Candeeiros, já que o seu ombroclima é subhúmido a húmido.

Em metade dos inventários verifica-se a ocorrência de espécies características da Ordem Pistacio-Rhamnetalia alaterni Rivas-Martínez 1975. Poderá indicar por vezes uma certa degradação especialmente no IF 3.

Os biótopos dos inventários realizados parecem ser mais húmidos 1 e 2, menos os 3 e 5 e mais frescos os 4 e 6; mais descalcificado parece ser o 6 .

Braun-Blanquet et. al (1956) consideraram no Arisaro-Quercetum broteroi as subassociações ericetosum, vincetosum e phillyretosum. Neste Parque Natural a subassociação mais próxima é, sem dúvida, a vincetosum.

Segundo Braun-Blanquet et. al (1956) a degradação dos carvalhais pertencentes ao Arisaro clusii-Quercetum broteroi pode conduzir a Melico minutae-Quercetum cocciferae Br.-Bl., P. Silva \& Rozeira 1956 (Espírito Sánto \& Lousã, 1988).

Por aplicação do método dos perfis ecológicos e da informação mútua aos inventários realizados em todos os tipos de vegetação do Parque Natural das Serras de Aire e Candeeiros verifica-se que as espécies mais sensíveis à dominância de Quercus faginea Lam. ssp. broteroi (P. Coutinho) A. Camus são: Aristolochia paucinervis Poumel, Cistus albidus, L., Brachipodum phoenicoides (L.) Roem. \& Sch. var. phoenicoides, Salvia sclareoides Brot., Crepis vesicaria L. ssp. haenseleri (DC.) P. D. Sell, Pteridium aquilinum (L.) Kuhn, Cistus salvifolius L., Vicia sativa L. ssp. nigra (L.) Ehrh., Daphne gnidium L., Erica scoparia L. ssp. scoparia, Urginea maritima (L.) Baker, Stachys germanica L. ssp. lusitanica Hoffmanns. \& Link, Geranium purpureum Vill., Rubus ulmifolius Schott, Geum sylvaticum Pourret, Plan- 
tago lanceolata L., Polypodium australe Fée, Lonicera etrusca G. Santi, Crataegus monogyna Jacq. ssp. brevispina (G. Kunze) Franco, Hyacinthoides hispanica (Miller) Rothm. e Euphorbia characias L. ssp. characias.

A G R A D E C I M E N T O. Ao Prof. S. Rivas-Martínez agradece-se os esclarecimentos prestados.

\section{B I B L I O G R A F I A}

ALBUQUERQUE, J.P.M. -1978- Carta pluviométrica analítica de Portugal pelo método de zonagem, E.A.N., INIA Oeiras.

AlCOFORADO, J.J., ALEGRIA, M.F., PEREIRA, A.P. \& SIRGADO, M. -1982Domínios bioclimáticos em Portugal definidos por comparaçãao dos índices de Gaussen e de Emberger, Cent. Est. Geogr., INIC, Lisboa.

BACOU, A.M. \& LEPART, J. -1984-Dispositif et mode d'utilisation en libre service de la Bibliothèque Infeco implantée sur microordinateur Corail en vue de la gestion et du traitement de fichiers de releves Phyto et Zoo-écologique, C.N.R.S.

BRAUN-BLANQUET, J. -1932- Plant. Sociology. The study of plant communities. McGraw Hill, New York.

BRAUN-BLANQUET, J., PINTO DA SILVA, A. \& ROZEIRA, A. -1956- Resultats de deux excursions géobotaniques à travers le Portugal Septentrional et Moyen. II. Agron. Lus., 18(3):167-235. Sacavém.

DAGET, P. \& GODRON, M. -1982- Analyse frequentielle de l'écologie des espèces dans les communautés. Ed. Masson. Paris.

ESPIRITO SANTO, M.D. \& LOUSÃ, M. -1981- A Flora do Parque Natural das Serras de Aire e Candeeiros, C.B.A.A.U.T.L., Lisboa.

ESPIRITO SANTO, M.D. \& LOUSÃ, M. -1988- Os carrascais do Parque Natural das Serras de Aire e Candeeiros. Symp. Bot. Pius Font $i$ Quer, Lleida.

FRANZI, A.V. -1984- Flora y vegetación vascular de la vertiente sur de la Sierra de Gata (Cáceres).Thes. Doct., Universidad de Salamanca.

GÉHU, J.M. \& RIVAS MARTINEZ, S. -1980- Notions fondamentales de Phytosociologie in Syntaxonomie. J. Cramer. Vaduz.

GODRON, M., DAGET, P., EMBERGER, L. LONG, G., LE FLOC, H.E., POISSONET, J., SAUVAGE, C. \& WACQUANT, J.P. -1968-Code pour le relevé méthodique de la végétation et du milieu (principes et transcription sur cartes perforées). C.N.R.S., Paris.

LEPART, J. -1982- Guide d'utilisation de quelques-uns des programmes de la chaine de traitement des données phyto-écologiques de l'Ecothéque Mediterrannéene (Document de travail). C.N.R.S. Montpellier.

MULLER, T. -1962- Die Saumgesellschaften der Klasse Trifolio-Geranietea sanguinei. Mitt. Florist. Soc. Arb., 9:95-140. Stolzenau.

RIVAS MARTINEZ, S. -1975- La vegetación de la Quercetea ilicis en España y Portugal. Anal. Inst. Bot. Cavanilles, 31(2):205-259. Madrid.

RIVAS MARTINEZ, S. -1987- Memoria del Mapa de Series de Vegetación de España, I.C.O.N.A., Madrid.

(Aceptado para su publicación el 5 de febrero de 1990)

Dirección de los autores: Departamento de Botânica, Instituto Superior de Agronomia, 1399 Lisboa Codex, Portugal. 
Quadro fitossociológico definitivo para os carvalhais de Quercus faginea Lam. ssp. broteroi (P. Coutinho) A. Camus do Parque Natural das Serras de Aire e Candeeiros

$\begin{array}{lcccccc}\text { N: de ordem } & 1 & 2 & 3 & 4 & 5 & 6 \\ \text { IF n: } & 195 & 197 & 204 & 212 & 208 & 213 \\ \text { Area mínima } & 100 & 100 & 20 & 20 & 10 & 10 \\ \text { Mês } & 4 & 4 & 5 & 5 & 5 & 5 \\ \text { Exp. } & \mathrm{E} & \mathrm{E} & \mathrm{W} & \mathrm{NW} & \mathrm{W} & \mathrm{N} \\ \text { Alt. (m) } & 375 & 375 & 175 & 200 & 200 & 420 \\ \text { Grau de cobertura (áryores) } & 80 & 90 & 80 & 70 & 80 & 60\end{array}$

\section{Características da Associaçao (Arisaro-Quercetum broteroi):}

Quercus faginea Lam.

ssp. broteroi (P. Coutinho) A. Camus

Diferenciais da subassociaçao vincetosum:

$\begin{array}{cccccc}5.5 & 5.5 & 4.5 & 4.4 & 5.5 & 1.1 \\ . & . & . & . & 1.2 & 1.1\end{array}$

Genista tournefortii Spach

Características da Aliança (Quercion broteroi):

Paeonia broteroi Boiss. \& Reuter

Arisarum vulgare Targ.-Tozz. ssp. vulgare

Hyacinthoides hispanica (Miller) Rothm.

Smilax aspera $\mathbf{L}$.

$1.1 \quad$ R.1 1.2

$+.1+.2$

Características de ordem e da classe: (Quercetalia ilicis, Quercetea ilicis):

Daphne gnidium L.

Ruscus aculeatus L.

Rubia peregrina $L$.

Lonicera etrusca G. Santi

Quercus suber $\mathrm{L}$.

Anemone palmata L.

Osyris alba $\mathbf{L}$.

Rosa sempervirens $\mathrm{L}$.

Pistacia lentiscus $\mathrm{L}$.

Pulicaria odora (L.) Reichemb.

Poligonatum odoratum (Miller) Druce

$\begin{array}{cccccc}1.1 & 1.1 & +.1 & . & +.1 & . \\ +.1 & . & 2.3 & . & +.1 & . \\ 2.1 & . & . & . & 1.2 & 1.1 \\ 1.1 & +.1 & +.1 & . & . & . \\ . & . & +.1 & . & . & 4.1 \\ +.1 & . & . & . & +.1 & . \\ . & . & . & +.1 & . & . \\ . & 1.1 & . & 1.1 & . & . \\ . & . & 2.2 & . & . & . \\ . & . & +.1 & . & . & . \\ . & . & 1.2 & . & . & .\end{array}$

Características das classes (Quercetea ilicis, Querco-Fagetea):

Geum sylvaticum Pourret

Aristolochia paucinervis Poumel

$\begin{array}{llllll}+.1 & 1.1 & +.2 & . & +.1 & 2.1\end{array}$

Rubus ulmifolius Schott

Luzula forsteri (Sm.) DC

Tammus communis $\mathrm{L}$.

Hedera helix L. ssp. canariensis (Willd.) Coutinho

$+.1 \quad 1.1$

Arum italicum Miller

Cephalanthera longifolia (Huds.) Fritsch

Asplenium onopteris L.

Ulmus minor Miller

$\begin{array}{llll}2.2 & +2 & 1.2 & 1.2\end{array}$

$\begin{array}{llllll}+.2 & +.2 & . & +.2 & . & 1.2\end{array}$

$\begin{array}{llllll}+.1 & +.1 & . & . & \text { R. }\end{array}$

Helleborus foetidus L

Frangula alnus Miller

$+.1 .2 .3 \quad$. 2.3.

$\begin{array}{lllll}+.1 & +.1 & . & . & . \\ +.1 & +.1 & . & .\end{array}$

$+.1+.1$

$+.1 \quad$.

Características da ordem (Pistacio-Rhamnetalia alaterni)

Quercus coccifera $\mathrm{L}$.

Rhamnus alaternus L.

$1.1 \quad 2.2 \quad \cdot \quad \cdot \quad \cdot$

Myrtus communis L.

Jasminum fruticans $\mathrm{L}$.

Características da classe (Calluno-Ulicetea):

Erica scoparia L.

Lithodora prostrata (Loisel) Griseb. ssp. prostrata

Genista triacanthos Brot.

$\cdot$

1.2 .

$\begin{array}{llllll}+.1 & . & 3.2 & . & . & 1.1\end{array}$

Características da classe e ordem (Trifolio-Geranietea sanguinei e Origanetalia):

Geranium purpureum Vill.

Stachys germanica L.

ssp. lusitanica (Hoffmanns. \& Link) Coutinho

$\begin{array}{llllll}+.1 & 1.1 & +.1 & +.1 & +.1 & +.\end{array}$

Salvia sclareoides Brot.

$\begin{array}{lllll}1.1 & +.1 & +.1 & +.1 & +.\end{array}$.

Clinopodium vulgare $\mathrm{L}$.

$\begin{array}{llll}1.1 & 1.1 & +.1 & +.1\end{array}$

Sedum forsteranum Sm.

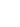
5 20 
Calamintha baetica Boiss. \& Reuter

Origanum virens Hoffmanns. \& Link

Rumex acetosa L.

Silene alba (Miller) E.H.L. Krause

ssp. divaricata (Reichenb.) Walters

\section{Companheiras:}

Vicia sativa L. ssp. nigra (L.) Ehrh.

Cistus salvifolius L.

Umbilicus rupestris (Salisb.) Dandy

Sanguisorba minor Scop.

Trifolium minor Scop.

Urginea maritima (L.) Baker

Silene patula Desf.

Ranunculus bulbosus L.ssp. adscendens (Brot.) Neves

Cytisus striatus (Hill) Rothm.

Ferula tingitana L.

Thapsia villosa L. var. platyphyllos Franco \& P. Silva

Plantago lanceolata L.

Bellis sylvestris Cyr.

Muscari comosum (L.) Miller

Allium roseum $\mathrm{L}$.

Brachypodium phoenicoides (L.) Roem \& Sch. var. phoenicoides

Carex flacca Schreber ssp. flacca

Asplenium trichomanes L.

Stellaria media (L.) Vill.

Cerastium glomeratum Thuill.

Ranunculus omiophyllus Ten.

Agrimonia eupatoria L.

Potentilla reptans L.

Ulex europaeus L. ssp. europaeus

Vicia lutea L. var. lutea

Lathyrus sylvestris $\mathrm{L}$.

Lathyrus aphaca L.

Trifolium pratense L.

Cistus albidus L.

Daucus muricatus (L.) L.

Cynoglossum creticum Miller

Calamintha baetica Boiss. \& Reuter

Cynara humilis L.

Rhagadiolus edulis Gaertner

Aetheorhiza bulbosa (L.) Cass.

Sonchus tenerrimus L.

Ornithogalum ortophyllum Ten.

ssp. baeticum (Boiss.) Zahar.

Dactylis glomerata L.

Anacamptis pyramidalis (L.) L.C. Rich

$\begin{array}{cccccc}. & . & . & . & 1.1 & +.1 \\ 1.1 & 1.1 & . & . & . & . \\ . & \text { R.1 } & . & . & . & .\end{array}$

$\begin{array}{cccccc}. & . & 1.1 & . & . & . \\ 2.1 & . & +.1 & 1.1 & 1.1 & +. \\ 2.2 & . & +.1 & 1.1 & 1.1 & +. \\ +.1 & 1.1 & . & +.1 & \mathrm{R} .1 & . \\ +.1 & +.1 & +.1 & . & . & 1.1 \\ . & +.1 & . & +.1 & 2.2 & +. \\ 1.1 & . & 1.1 & +.1 & +.1 & . \\ . & . & +.2 & 1.2 & +.2 & . \\ +.1 & 1.1 & +.2 & . & . & . \\ . & . & 1.2 & 3.3 & +.2 & . \\ . & 1.1 & . & \mathrm{R} .1 & . & +. \\ . & +.1 & 1.1 & +.1 & . & . \\ . & . & +.2 & 1.1 & +.1 & . \\ 1.1 & +.1 & +.1 & . & . & . \\ +.1 & +.1 & . & +.1 & . & . \\ +.1 & +.1 & . & \mathrm{R} .1 & . & .\end{array}$

\begin{tabular}{|c|c|c|c|c|}
\hline 2.2 & . & 1.2 & . & 1.2 \\
\hline 1.2 & . & . & +.2 & 1.2 \\
\hline+.1 & . & . & . & . \\
\hline 1.1 & +.1 & . & . & . \\
\hline+.1 & . & +.2 & . & . \\
\hline . & . & . & +.1 & . \\
\hline+.1 & 1.1 & . & & . \\
\hline . & 1.1 & . & . & +.1 \\
\hline . & . & 1.2 & . & \\
\hline . & 1.1 & . & 1.1 & . \\
\hline 1.1 & . & +.1 & . & . \\
\hline . & +.1 & . & . & 2.1 \\
\hline . & 1.3 & . & . & 2.2 \\
\hline . & 1.1 & . & 1.2 & . \\
\hline . & . & . & +.1 & . \\
\hline+.1 & +.1 & . & . & . \\
\hline . & . & . & . & 1.1 \\
\hline+.1 & . & +.1 & . & . \\
\hline 1.1 & 1.2 & . & . & . \\
\hline . & 1.1 & . & +.1 & . \\
\hline . & +.1 & . & . & R.1 \\
\hline 1 & +.1 & . & . & . \\
\hline & . & . & 3.2 & . \\
\hline & +.1 & . & . & R.1 \\
\hline
\end{tabular}

\section{Localização dos inventários fitossociológicos}

1- Porto de Mós, Arrimal, Arrabal. 2- Porto de Mós, Serro Ventoso, no vale abaixo de Malhadais.

3- Alcobaça, Aljubarrota, Casal do Doutor. 4- Por de Mós, Pedreiras, Vale Travelho.

5- Porto do Mós, Alqueidão da Serra, Cabeço do Moínho Novo. 6- Porto de Mós, S. Pedro, Pragais. 